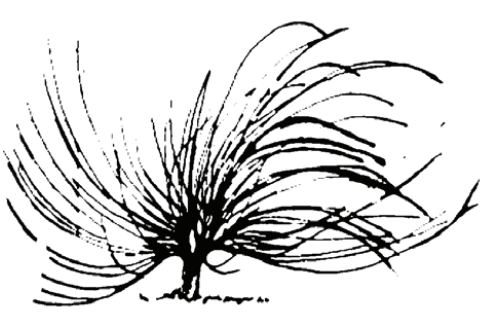

\title{
Aprendizaje entre Iguales y Aprendizaje Cooperativo: Principios Psicopedagógicos y Métodos de Enseñanza
}

\author{
Giovanni Sánchez Chacón ${ }^{1}$ \\ Universidad Nacional \\ Heredia, Costa Rica \\ giovanni.sanchez.ch@gmail.com
}

\begin{abstract}
Resumen
En el presente artículo, se presenta una revisión de la literatura sobre los fundamentos teóricos, estructuras de organización social y dimensiones del aprendizaje entre iguales, posteriormente se desarrollan temáticas en torno al aprendizaje cooperativo y sus respectivas condiciones para su implementación en las aulas, a través de los principales métodos señalados por la bibliografía especializada.
\end{abstract}

\section{(c) (i) $(2)(9$}

Recibido: 5 de diciembre de 2014-Aprobado: 26 de noviembre de 2015

1 Doctor en Psicología de la Educación, Cultura y Sistemas Semióticos. Universidad Autónoma de Barcelona, España. Posgrado en Psicología Evolutiva y de la Educación. Universidad Autónoma de Barcelona, España. Máster en Musicoterapia. Instituto Superior de Estudios Psicológicos, Barcelona, España. Máster en Psicopedagogía. Universidad Estatal a Distancia, UNED, Costa Rica. Licenciado en Ciencias de la Educación con énfasis en Didáctica de la Música. Universidad Nacional, Costa Rica. Bachiller en Música con 
Palabras clave: aprendizaje cooperativo, aprendizaje entre iguales, tutoría entre iguales, cooperación, colaboración, competición, gestión social del aula

\begin{abstract}
This article shows a literature review about the theoretical fundamentals, structures of social organization and dimensions of peer learning, which will be then related to cooperative learning concepts and the conditions for its implementation in the classroom through the main methods identified in the specialized literature.
\end{abstract}

Keywords: Cooperative learning, peer learning, peer tutoring, cooperation, collaboration, competition, social classroom management

\title{
Introducción
}

El presente artículo tiene como propósito el desarrollo de una revisión de la literatura especializada, (Johnson; Johnson; Holube, 1999); (Durán y Vidal 2004); (Topping, 2005); (Sánchez, 2009); (Durán, 2014), que a nivel general, ha establecido principios y pautas para la comprensión del marco teórico y metodológico del aprendizaje entre iguales y el aprendizaje cooperativo y sus prácticas intencionales en el aula. Dentro de este marco conceptual, no se concibe el uso del aprendizaje entre iguales desde una perspectiva instrumentalizada, sino que se establecen principios y pautas para que el docente, a través de una permanente autoevaluación de su práctica, recurra a decisiones de carácter estratégico, que garantice un uso consciente y deliberado de estos principios en su práctica cotidiana. Con el propósito de orientar la escritura del presente artículo, se plantean los siguientes objetivos:

1. Analizar los fundamentos teóricos del aprendizaje entre iguales, como marco teórico general, que da origen a nuevas formas de interacción en el aula.

2. Determinar las implicaciones pedagógicas del Aprendizaje Cooperativo como forma de gestión social del aula. 
3. Conocer los métodos de aprendizaje cooperativo más empleados por la investigación especializada (Johnson, Johnson, Holubec, 1999; Durán y Vidal, 2004; Topping, 2005; Sánchez, 2009; Durán, 2014) y sus posibles usos en situaciones de enseñanza y aprendizaje.

\section{Fundamentos teóricos del aprendizaje entre iguales}

El aprendizaje entre iguales, como forma de gestión social del aula y metodología de enseñanza, aún no culmina su proceso de redefinición epistemológica, aún así, diversos autores han aportado argumentos que permiten comprender con mayor claridad las posibles bases conceptuales que fundamentan dichas prácticas. De acuerdo con Ferreiro y Calderón (2000), el aprendizaje entre iguales integra el aporte de ciertas teorías relacionadas con el desarrollo organizacional, la motivación, el desarrollo cognitivo y el desarrollo de la personalidad. En esta misma línea, Melero y Fernández (1995), haciendo referencia al aporte del Equipo de Psicología Social de la Escuela de Ginebra, con una tendencia mayormente socio-cognitiva, conciben el aprendizaje entre iguales como un elemento indispensable para el desequilibrio Piagetiano, atribuyéndole un mayor protagonismo a los factores de carácter social en la construcción de conocimientos.

Otra de las ideas que nos ayudan a comprender el funcionamiento del aprendizaje entre iguales, es la teoría sociocultural desarrollada a partir de los trabajos de Vygotsky, la cual otorga a la interacción social una importancia relevante de cara a la construcción social de la mente humana. Esta situación se puede comprender con mayor claridad en el concepto Vygotskyano de Zona de Desarrollo Próximo, el cual es representado mediante el nivel de desarrollo real del niño, a partir de la resolución independiente de problemas y el nivel más elevado de desarrollo potencial, determinado por la resolución de situaciones problema bajo la tutela de un adulto o en colaboración co cncon $\mathrm{n}$ sus iguales más capacitados (Wertsch, 1988). De acuerdo con lo anterior, ciertos procesos cognitivos vinculados con el aprendizaje tienen su origen en situaciones sociales, por lo cual se deriva lo que Vygostky llamó la Ley Genética del Desarrollo Cultural, que establece que toda función en el desarrollo cultural del niño aparece dos veces y en dos planos distintos: 
primero en el nivel interpsicológico o social y luego en el intrapsicológico o individual.

Este proceso mediado que va del plano interpsicológico al intrapsicológico, en la perspectiva de Vygostky se le conoce como proceso de internalización, el cual de acuerdo con Ferreiro y Calderón (2001), sigue una ruta de mediación social que está caracterizada en primera instancia por la no regulación, luego la regulación a nivel de grupo y por último la autorregulación por parte del sujeto (intrapsicológico), al hacer suyo lo externo. Por consiguiente, es claro que en todo proceso de aprendizaje se producen una serie de mecanismos dentro del individuo, los cuales se activan mediante la interacción de un aprendíz con otros, facilitando la adquisición de conocimientos y el desarrollo de habilidades actitudes y valores.

Lo anterior sugiere que en las diversas situaciones de aprendizaje entre iguales, los estudiantes tomarían el papel de mediadores en el plano interpsicológico, quienes tienen la posibilidad de ofrecerse ayudas pedagógicas más ajustadas a sus necesidades, a través de la construcción de Zonas de Desarrollo Próximo, que permitan a los sujetos hacer suyo el conocimiento mediante el proceso de internalización, de esta manera, autorregular el conocimiento adquirido. Queda claro que dentro de este marco de interacción, los estudiantes aprenden y construyen el conocimiento de manera conjunta, a través procesos de andamiaje provistos por sus iguales (Durán y Monereo 2008).

Además de las anteriores aportaciones, existen algunos señalamientos en torno a modelos de aprendizaje entre iguales (Grannot,1993; Murray, 2001; Slavin, 1996), los cuales aún no tienen establecido un fundamento teórico consolidado. Sin embargo, de acuerdo con (Tooping, 2005 y Duran y Monereo, 2008) se han identificado ciertos mecanismos que podrían explicarnos la efectividad atribuida al aprendizaje entre iguales. Entre estos podemos citar las aportaciones teóricas provenientes del constructivismo social, las cuales atribuyen a la negociación de significados a través de un tipo de interacción basada en el dialogo, como uno de los mecanismos principales para la interiorización de los conocimientos compartidos en una situación de interacción entre iguales.

Además de las anteriores aportaciones, la investigación especializada, ha señalado diversos mecanismos interpsicológicos, (Colomina y Onrubia 2005), que usualmente suelen activarse en la interacción y aprendizaje entre iguales y los cuales se comentan a continuación. 
Aprendizaje entre Iguales y Aprendizaje Cooperativo:

\section{Mecanismos interpsicológicos en el aprendizaje entre iguales}

Uno de los factores que permite una mejor comprensión acerca de las formas de interacción subyacentes en el aprendizaje entre iguales, podría atribuírsele al estudio de un conjunto de mecanismos interpsicológicos que surgen espontáneamente en formas similares de interacción, los cuales ejercen una influencia favorable en los resultados de aprendizaje por parte de los alumnos. Al respecto, Colomina y Onrubia (2005) hacen mención a dichos mecanismos, los cuales podrían facilitar al estudiante la construcción del conocimiento, entre estos tenemos:

a. El conflicto entre puntos de vista moderadamente divergentes: este señala que en situaciones de interacción entre estudiantes, suelen surgir puntos de vista divergentes en relación con la tarea o actividad que se ejecuta. Esta situación de conflicto generada entre los estudiantes, puede favorecer a la vez el replanteamiento y revisión de los propios conceptos para una mayor organización de ellos, en torno a la tarea que se ejecuta.

b. Regulación mutua a través del lenguaje: esta fase está marcada por los diversos usos semióticos del habla como instrumento mediador que los estudiantes emplean en los procesos de interacción, lo cual se da con el propósito de regular la comunicación entre ellos y orientar el proceso de construcción compartida de conocimientos.

c. El apoyo a la atribución del sentido de aprendizaje: la interacción entre alumnos como ya hemos visto, no solo promueve la construcción de significados, sino también una atribución positiva del sentido de aprendizaje, ya que en este proceso, por lo general, surgen también mecanismos de carácter motivacional y afectivo, que podría contribuir a la atribución de un sentido práctico a la tarea y propiciar una adecuada actuación cooperativa y más solidaria entre los estudiantes que conforman los grupos de trabajo.

Por su parte, otras aportaciones señaladas por Topping, (2005) pretenden hacer un intento de definición teórica del aprendizaje entre iguales, al hacer una revisión teórica en torno a las temáticas en que se han centrado las investigaciones en este ámbito en los últimos años. De acuerdo con el mismo autor, la mayoría de los estudios realizados hasta 
el momento en esta temática se podrían vincular en cinco categorías entre las cuales se distinguen:

a. Organización y Compromiso: esta tiene que ver con las características organizacionales y estructurales que adoptan las interacciones entre los estudiantes, hace referencia a la necesidad de que estos elaboren metas y planes que faciliten el trabajo en conjunto y la individualización del aprendizaje. Lo anterior hace que se desarrolle un mayor compromiso mutuo hacia la tarea planteada, estimulándoles a adoptar nuevas formas de interacción.

b. Conflicto Cognitivo: esta posee relación con el conflicto socio-cognitivo relacionado con la escuela Piagetiana. Desde esta perspectiva, el aprendizaje se produce cuando los estudiantes tienen la oportunidad de confrontar sus puntos de vistas moderadamente divergentes y reflexionan en conjunto, teniendo así la posibilidad de modificar sus propias ideas, creencias y formas de aprendizaje.

c. Andamiaje y gestión del error: este posee relación con el concepto de Andamiaje y Zona de Desarrollo Próximo de la tradición Vygotskiana. Con el proceso de andamiaje, los estudiantes más aventajados brindan ayudas pedagógicas precisas y ajustadas al resto de sus compañeros, con el propósito de introducirse en la Zona de Desarrollo Próximo de ellos.

d. Comunicación: esta también requiere de la necesidad de que los estudiantes posean habilidades de comunicación, se propone la idea de que uno o varios conceptos pueden llegar a reafirmase o a entenderse con mayor profundidad, en el momento en que estos son explicados a otros sujetos, los cuales escuchando, cuestionando, especulando, hipotetizando y discutiendo en torno a una temática específica, podrían generar el desarrollo de habilidades de comunicación.

e. Afecto: los trabajos vinculados a esta categoría apuntan a la buena relación que debe presentarse entre los estudiantes que trabajan en conjunto, en donde no se distinga una posición autoritaria por parte de ningún estudiante ni del tutor y una buena actitud por parte de este, basada en el entusiasmo, seguridad y comprensión, que favorezca la auto-confianza de los estudiantes tutorados. 
Aprendizaje entre Iguales y Aprendizaje Cooperativo:

Además un ambiente de lealtad y responsabilidad, podría ayudar a mantener la motivación de los estudiantes hacia la tarea.

Luego de reflexionar en torno a estos argumentos que permiten una comprensión más profunda acerca del funcionamiento del aprendizaje entre iguales, a continuación y de acuerdo con la investigación especializada (Johnson, Johnson, Holubec, 1999; Durán y Vidal 2004; Topping, 2005), se exponen las posibles estructuras de gestión social del aula que usualmente tienen lugar en situaciones de aprendizaje entre iguales.

\section{Estructuras de gestión social del aula en el aprendizaje entre iguales}

Antes de profundizar en el estudio de esta temática, es importante tener claro algunos conceptos, tales como el aprendizaje entre iguales y aprendizaje cooperativo, sus diferencias, características de organización social, formas de estructuración de las actividades y tipos de interacción.

De acuerdo con (Deutsch 1949; Johnson, Johson y Holubec 1999) y en trabajos más recientes (Pujolás, 2007), dentro del aprendizaje entre iguales podemos distinguir tres formas de organización social del aula, las cuales a pesar de estar orientadas al trabajo en grupo, persiguen distintos objetivos, entre estas tenemos:

a. Situaciones cooperativas: estas se refieren a un tipo de estructura cooperativa de las actividades que se desarrollan en una clase, la cual lleva a los alumnos a contar unos con otros, a colaborar y a ayudarse mutuamente a lo largo del desarrollo de la actividad, Pujolás y Lago (2007). En estas los propósitos de los participantes están muy relacionados, de tal forma, que cada uno alcanza sus objetivos en la medida que cada miembro consigue alcanzar los suyos, o sea, que los resultados que cada uno consigue, benefician a los demás. Monereo, Pozo y Castelló (2001) catalogan este tipo de situaciones, como una dinámica de trabajo que favorece la práctica guiada, ya que mediante su implementación se podría incrementar la enseñanza de estrategias de aprendizaje en actividades donde un grupo de estudiantes resuelven una tarea colaborativamente.

b. Situaciones competitivas: a diferencia de la anterior, en esta no todos pueden ganar. Estas se caracterizan por el hecho de que 
los estudiantes alcanzan sus objetivos, en la medida en que los demás no alcancen los propios. De acuerdo con Pujolás y Lago (2007), las estructuras competitivas de la actividad en una clase, por lo general, suelen llevar a los alumnos a rivalizar entre ellos por ser el primero que acaba la tarea o el que sabe más lo que el profesor les enseña. Los mismos autores manifiestan que en estas situaciones los estudiantes tienden a ocultar información, a guardar celosamente las respuestas de ciertas preguntas, la solución de un problema o la manera de resolverlo.

c. Situaciones individualistas: estas se presentan cuando cada estudiante obtiene sus propios resultados, sin que se perciban relaciones entre estos y los de los demás.

Como se ha expuesto, los tipos de interacción que pueden suscitarse entre estudiantes en una clase son variadas y persiguen distintos objetivos. Por lo general, la enseñanza tradicional ha priorizado la estructura competitiva y la individual, dando menos cabida a la estructura cooperativa.

Así como dentro del aprendizaje existen estructuras de gestión social del aula, también en el aprendizaje entre iguales se pueden identificar formatos o dimensiones sobre las que se pueden establecer situaciones de aprendizaje entre iguales, las cuales se exponen a continuación.

\section{Dimensiones del aprendizaje entre iguales}

En cuanto a las formas de organización de la actividad por parte de los alumnos en situaciones cooperativas y haciendo referencia a los principios señalados por Damon y Phelps (1989), se identifican las siguientes:

a. Colaboración entre iguales: esta se da cuando dos o más alumnos de niveles similares trabajan en forma colaborativa de una forma constante, en vías de la resolución de una tarea.

b. Aprendizaje cooperativo: consiste en la realización por parte de los estudiantes de tareas o actividades preestablecidas, en la que se presentan formas de interacción que permiten la discusión, planificación y distribución de las responsabilidades.

c. Tutoría entre iguales: en esta, un alumno considerado como experto en algún campo o contenido específico instruye a otro 
considerado como menos experto o novato. En esta también se da un cierto nivel de planificación y distribución de las responsabilidades tanto en el alumno tutor como en el tutorado.

De lo comentado, se infiere que el aprendizaje entre iguales posee distintas estructuras de organización, las cuales van desde la colaboración, en la que tan solo se plantean agrupamientos de estudiantes con el propósito de resolver en conjunto ciertas tareas de aprendizaje, hasta la cooperación y la tutoría, las cuales suelen ser planificadas de una forma más intencional en donde, por lo general, las actividades han sido previamente establecidas y se distingue un mayor nivel de distribución de las responsabilidades.

Dentro del aprendizaje entre iguales, es oportuno rescatar el papel de las situaciones cooperativas ya que por los fines que estas persiguen, el tipo de interdependencia que se desarrolla entre los estudiantes y sus estructuras de organización social, estas suelen desarrollar patrones de interacción muy adecuados que favorecen el buen aprendizaje de los alumnos.

\section{Fundamentos teóricos del Aprendizaje Cooperativo}

El aprendizaje cooperativo representa una forma de organización social del aula, situada dentro del marco conceptual del aprendizaje entre iguales y caracterizada por el tipo de interdependencia que se fomenta entre los compañeros pertenecientes a un grupo de trabajo.

En la actualidad, diversos autores proponen una serie de conceptualizaciones en torno al aprendizaje cooperativo. Al respecto Johnson, Johnson y Holubec (1999), lo definen como el empleo didáctico de grupos reducidos en los que los alumnos trabajan juntos para maximizar su propio aprendizaje y el de los demás. Por otra parte, Topping (2005) lo concibe como un proceso de adquisición de conocimientos y habilidades, mediante la ayuda activa y mantenida de otro u otros compañeros de estatus similares, en donde usualmente se ejecutan tareas de aprendizaje en un contexto de trabajo en equipo, en el cual los esfuerzos de todos los participantes se combinan con el propósito de alcanzar un objetivo en común, conocido y compartidos por todos sus miembros.

Debido al nivel de planificación deliberada que el aprendizaje cooperativo comporta, este en ningún caso deberá concebirse como un 
simple agrupamiento al azar de dos o más estudiantes. De esta manera, sería imposible lograr los beneficios académicos que a esta estrategia se le atribuyen. Para obtener mayores ventajas de su implementación, diversos autores (Cohen, Kulik y Kulik 1982; Hairul y Joyce 2005 o Duran y Vidal 2004) sugieren optar por la planificación de sesiones de trabajo de manera estructurada, por lo que cada estudiante, independientemente de la forma de organización cooperativa con que se desee trabajar, sepa en todo momento cuales son las tareas a seguir, las cuales se desprenden del rol que les ha sido asignado.

Aún así, es importante que exista una cierta libertad en los roles que cada sujeto desempeña y no se presente una sobreestructuración de la sesiones de trabajo, que pueda restar creatividad y espontaneidad a la interacción de los estudiantes. Por ello, en el aprendizaje cooperativo, suele primar un marco de relación entre los participantes del equipo altamente estructurado por el profesor, pero a su vez que permita una progresiva apropiación de este por parte de los alumnos, modificando y ajustando esa estructura interactiva a las necesidades y condiciones del contexto de aprendizaje (Sánchez, 2009).

\section{Condiciones del aprendizaje cooperativo}

Como se ha comentado, el aprendizaje cooperativo no consiste en una simple agrupación de estudiantes en torno a una tarea de aprendizaje, ya que de esta manera no se podrían obtener los beneficios de carácter instruccional que a esta estrategia se le atribuyen. De acuerdo con Johnson, Johnson y Holubec, (1999), existen una serie de condiciones que puestas en marcha, generan mejores resultados en el funcionamiento de situaciones de cooperación en las aulas, entre estas podemos mencionar:

a. Interdependencia positiva: esta es considerada por los mismos autores como la clave para una cooperación efectiva, la cual consiste en que el éxito de cada uno de los miembros representa también el éxito de todo el equipo. En esta etapa, debe haber una certeza de que todos los miembros del grupo aprendan y los continuos refuerzos que el estudiante recibe son dados mediante un reconocimiento grupal.

b. Interacciones cara a cara: en esta se fomentan todas las situaciones de interacción que generen actuaciones interpersonales de 
colaboración y estímulos positivos entre los miembros del equipo. En cuanto a la cantidad de integrantes por cada agrupación, algunos autores sugieren limitarlos a cuatro estudiantes para un mejor establecimiento de las condiciones descritas.

c. Responsabilidad individual: esta intenta suprimir la difusión de responsabilidades, para esto algunos autores recomiendan la evaluación individual, la elección aleatoria del líder del grupo o la incorporación de informes personales de trabajo, con la nota de equipo.

d. Habilidades sociales: para que exista una buena comunicación en el entorno del grupo, es necesario que cada uno de sus miembros conozcan y pongan en práctica ciertas habilidades imprescindibles para el establecimiento de procesos de cooperación, entre estas podríamos citar: actitud de participación, fluidez en la comunicación, resolución constructiva de conflictos, aceptación de los puntos de vista de sus compañeros etc.

e. Autorreflexión de grupo: en este proceso, los miembros del grupo, proceden a reflexionar de manera colectiva en relación con el trabajo que se les propone. De esta manera, toman decisiones pertinentes que beneficiarán este proceso y proponen, a la vez, acciones a tomar en cuenta para mejorar su práctica en futuras sesiones.

Como hemos visto, el trabajo cooperativo que se establece en situaciones de aprendizaje entre iguales requiere de una serie de requisitos, los cuales generan las condiciones que favorecen el éxito de este tipo de actividades. A nivel general, la eficacia atribuida al aprendizaje cooperativo, suele estar íntimamente vinculada con el nivel de compromiso e interdependencia que se establece entre los participantes de un equipo de trabajo, como resultado del tipo de estructuración de la interacción, la cual ha sido planificada deliberada y estratégicamente por parte del docente.

No resulta pues sencillo crear condiciones adecuadas para la implementación exitosa del aprendizaje cooperativo, pero la literatura especializada, ha propuesto ciertos métodos de aprendizaje, que ajustados a las condiciones del contexto de enseñanza y a las particularidades propias de los contenidos a enseñar, podrían resultar una ayuda eficaz en el diseño de situaciones de aprendizaje cooperativo en las aulas. 


\section{Métodos de Aprendizaje Cooperativo}

Duran y Monereo (2002) definen los métodos de aprendizaje cooperativo como una metodología que convierte la heterogeneidad, vista como las diferencias existentes entre los alumnos, en un elemento positivo que facilita el aprendizaje.

La relevancia y diversidad de métodos de aprendizaje cooperativo en la actualidad, ha hecho necesario el intento de múltiples clasificaciones de ellos (Ovejero 1991; Sharan, 1994; Slavin, 1995; Pujolás 2003). Estos se han clasificado entre otras cosas en función de la responsabilidad individual, del agrupamiento del alumnado, del tipo de interdependencia, objetivos del grupo, entrenamiento y formación previa en habilidades sociales, aplicación en diferentes áreas curriculares, estructura de grupo y adaptación a las necesidades individuales (Davison, 1994; Slavin, 1995; Fernández 2007).

Actualmente, se pueden distinguir, por un lado ciertas técnicas y formatos muy sencillos, que promueven el aprendizaje entre iguales en situaciones temporalmente breves y sin necesidad de un uso sistemático, y y por otro, algunos métodos de aprendizaje cooperativo, entendidos como diseños didácticos algo más complejos que pretenden ayudar a crear situaciones cooperativas más dilatadas en el tiempo y exigentes en cuanto a resultados de aprendizaje.

Siendo innecesario presentar un inventario general de técnicas y métodos de aprendizaje cooperativo, a continuación se exponen, a titulo ilustrativo, algunos ejemplos de las metodologías más citadas por la investigación especializada.

\section{Tutoría entre iguales}

La tutoría, como una de las dimensiones metodológicas atribuidas al aprendizaje entre iguales, representa un método de aprendizaje cooperativo basado en la creación de parejas de alumnos con una relación asimétrica en cuanto a nivel de competencia y unos objetivos en común conocidos y compartidos por todos los miembros del equipo, los cuales se consiguen a través de un marco de relación exteriormente planificado (Duran y Vidal, 2004). La tutoría entre iguales no sólo beneficia al alumno tutorado, sino también al alumno tutor, el cual tiene la posibilidad de aprender mientras enseña a su compañero (Topping, 1996). Para ello, 
el docente optará por un marco de interacción altamente estructurado, a su vez, permite una progresiva apropiación de este por parte de los alumnos, modificando y ajustando esa estructura interactiva según las necesidades y condiciones del contexto de aprendizaje.

Los formatos de tutoría entre iguales pueden establecerse de muchas formas distintas. Así, en función de la edad y de la continuidad de los roles, se puede distinguir entre tutoría entre alumnos de distinta edad y de la misma edad. Esta última puede incluir situaciones de tutoría recíproca, en donde los estudiantes alternan el rol de tutor en cada sesión de trabajo.

Cabe destacar, que en la tutoría entre iguales, los beneficios no solo los obtienen los estudiantes tutelados. La literatura especializada (Durán y Sánchez, 2012); (Durán, 2014), hace hincapié en los beneficios académicos que gozan también los estudiantes que desempeñan la función de tutor. Los estudios disponibles exponen que, en determinadas condiciones, la actividad de enseñar comporta oportunidades de aprendizaje para quien la desempeña, (Durán, 2014).

\section{Puzzle (Rompecabezas)}

En este método es indispensable dos estilos de agrupamientos: el equipo base (heterogéneo) y el de expertos (homogéneo). El proceso se desarrolla en los siguientes pasos:

a. Se asigna de manera aleatoria los estudiantes en el grupo base, al cual se le encomienda la resolución de una actividad en particular, posteriormente, se le asigna a cada miembro un "subtema" clave para la resolución de dicha actividad, en el cual tendrán que convertirse en expertos.

b. Los estudiantes pasan del grupo base al grupo de expertos, en este todos los miembros tienen asignado el mismo tema en el cual tendrán que especializarse.

c. Posteriormente, se regresa al grupo base, en donde los estudiantes deberán exponer la parte de los conocimientos (equivale a una pieza del puzzle), que han adquirido en actividades resueltas en el grupo de expertos. Esta estructura permite que el aporte de cada miembro del equipo base se vuelva indispensable para la resolución de la actividad, la cual es evaluada mediante una nota de equipo. 


\section{Torneos de equipos y juegos}

Consiste en torneos académicos, en donde los estudiantes compiten como representantes de sus equipos. Cada miembro del equipo se ubica en un grupo homogéneo con el que competirá en la respuesta de preguntas, posteriormente, regresa al equipo original y aporta a este los puntos que ha ganado. En este caso, también el reconocimiento es de equipo y la puntuación de cada estudiante servirá para crear el grupo homogéneo de competición de la siguiente sesión de preguntas.

\section{Controversia constructiva}

Esta metodología, creada por los hermanos Johnson, académicos e investigadores de la universidad de Minnesota, consiste en la creación de controversias o incidentes críticos entre los estudiantes de un equipo, con el propósito de que ellos puedan llegar a establecer un acuerdo mutuo, al ofrecerles la oportunidad de acceder al punto de vista de cada uno de sus compañeros con respecto a dicha controversia.

En primera instancia, cada estudiante de manera individual investiga y prepara su posición, defendiéndola oralmente y teniendo en cuenta la perspectiva del resto de sus compañeros, para posteriormente integrar la información en una propuesta común.

\section{Enseñanza recíproca}

Este método, pensado para la resolución de actividades de comprensión lectora, ha sido desarrollado por Palincsar y Brown (1984). En este cada estudiante es responsable de la realización de una tarea distinta, relacionada con alguna operación cognitiva necesaria para la comprensión lectora. Con el propósito de llevar a cabo una tarea común, cada miembro del grupo realiza una función distinta, pero complementaria a la del resto de sus compañeros en donde se les enseña a cada integrante a cumplir su función cuando le corresponda su turno.

En este caso, se recomiendan equipos de máximo cuatro estudiantes, en los que se distribuyen sus roles de la siguiente manera:

1. Un estudiante lee el texto.

2. Otro simultáneamente va tomando apuntes de las ideas principales. 
3. El siguiente redacta preguntas o interrogantes interesantes que se desprenden del tema.

4. El último intentará anticipar las posibles temáticas que el autor tratará en las siguientes páginas o capítulos, así como el eventual desarrollo de los acontecimientos que en la lectura se narran.

Una vez terminado este proceso, los estudiantes discuten acerca del tema de la lectura, en función del rol que les fue asignado. Esta dinámica se realizará por cada página o capítulo que los estudiantes lean, esta decisión dependerá de las características y condiciones de los textos con los que se esté trabajando.

\section{Investigación de Grupo}

De acuerdo con Echeita y Martín (1990), este método podría ser el más complejo y el que mejor responde a la filosofía de los grupos cooperativos, ya que aporta una mayor variedad de experiencias de aprendizaje que las restantes técnicas, más orientadas a la adquisición de conocimientos y destrezas.

En este método, el grupo-clase trabaja como una comunidad de investigación, en torno a un tema específico, el cual es dividido en distintos subtemas o líneas de investigación, los cuales son asignados a diversos subgrupos. Al final del trabajo, cada subgrupo socializará sus resultados con el resto de la clase, a la vez, ofrecerá una reflexión acerca del trabajo en equipo efectuado.

\section{Pensar, aparejarse y compartir}

Esta dinámica suele ser muy práctica a la hora de integrarla como componente de otros métodos que se apliquen a largo plazo. Para llevarlo a la práctica, han de seguirse los siguientes pasos:

1. Los alumnos han de reflexionar en forma individual acerca de las principales demandas que giran en relación con el tema que plantea el profesor.

2. Posteriormente (unos pocos minutos después), cada estudiante se empareja con otro compañero con el propósito de 
discutir y comparar las conclusiones a las cuales llegaron ambos individualmente.

3. Después, los estudiantes comparten sus ideas con el resto de la clase. En este sentido, es importante delimitar el tiempo para cada etapa de la actividad y acordar señales (un sonido o una señal manual) para el paso a la siguiente fase.

Esta técnica presenta las siguientes ventajas:

1. Todos los estudiantes poseen mayor tiempo para pensar.

2. Es efectivo para todos los niveles educativos y tamaños de clases.

3. Muchos estudiantes, por lo general, que no tengan éxito al contestar alguna pregunta planteada por el profesor, tienen la oportunidad de ensayar las respuestas mentalmente.

4. Trabajar el tema con un compañero antes de tener que exponerlo al resto de la clase, podría contribuir al desarrollo de una mayor seguridad por parte del estudiante.

5. Como resultado, más estudiantes podrían estar dispuestos a participar y la calidad de sus respuestas probablemente mejoraran.

\section{Dentro y fuera del círculo}

Este tipo de estructura cooperativa requiere del uso de dos círculos concéntricos en el que cada estudiante debe sentarse mirando a otro compañero del otro círculo. Después, cada alumno escucha la pregunta realizada por el profesor y trabaja con el compañero que tiene en frente con el propósito de hallar la respuesta correcta.

Posteriormente a la discusión del grupo, se verifican las respuestas correctas y el círculo exterior se mueve a una posición hacia la derecha, de tal forma, que los estudiantes queden con otra pareja para la pregunta siguiente.

De acuerdo con los autores, esta estructura cooperativa funciona mejor en tareas que implican respuestas cortas. Uno de los beneficios de esta dinámica, consiste en que los estudiantes pueden interactuar con una gran variedad de compañeros. 


\section{Cabezas juntas y numeradas}

Esta dinámica pone en tensión la interdependencia positiva de los miembros del grupo. Los pasos a seguir para su desarrollo son los siguientes:

1. Se asigna un número a cada estudiante del grupo.

2. Se presenta la tarea de aprendizaje a desarrollar.

3. Los estudiantes se entrenan los unos a los otros sobre el material que han de aprender, de tal manera, que cualquiera pueda representar el grupo si el profesor selecciona su número durante el tiempo de respuesta.

Esta dinámica funciona particularmente bien en tareas instruccionales en las que los estudiantes investigan muchas facetas de un problema.

Como hemos visto, por la dinámica de organización de la interacción que estos formatos exigen, dichas metodologías, estratégicamente planificadas y gestionadas, permitirían abordar grandes cantidades de conocimientos en menor tiempo, si lo contrastamos con el uso de métodos expositivos y tradicionales de enseñanza. Lo anteriormente citado, nos lleva a continuación a plantear algunas conclusiones generales de lo expuesto hasta este punto.

\section{Conclusiones}

Las conclusiones del presente artículo se establecen en función de los objetivos que orientan su desarrollo, los cuales se expusieron en el capítulo inicial de introducción.

Con respecto al primer objetivo, que hace referencia al análisis de los fundamentos teóricos del aprendizaje entre iguales, como marco teórico general y que da origen a nuevas formas de interacción en el aula, podemos concluir lo siguiente:

a. $\quad \mathrm{Al}$ intentar definir y analizar ciertos conceptos propios del aprendizaje entre iguales, vinculados a sus mecanismos interpsicológicos, a las estructuras de gestión social del aula y sus respectivas dimensiones, se puede obtener una mayor comprensión de los dispositivos de carácter comunicativo, semiótico y psicológico 
que entran en juego en situaciones de interacción entre iguales y como ciertas iniciativas pedagógicas vinculadas a este paradigma, podrían surgir un amplio repertorio de estrategias de enseñanza eficaces, para ser empleadas tanto en educación formal como no formal y en cualquier ámbito del conocimiento.

Con respecto al objetivo número dos del presente artículo, el cual se propone determinar las implicaciones pedagógicas del aprendizaje cooperativo como forma de gestión social del aula, se concluye lo siguiente:

a. El aprendizaje cooperativo, como metodología de enseñanza catalogada dentro del marco del aprendizaje entre iguales, suele emplearse con mayor fundamento, en tareas y actividades académicas que requieren un alto nivel de trabajo en equipo, donde todos sus miembros poseen roles de trabajo concretos, los cuales ponen al servicio de los objetivos que orientan la actividad cooperativa.

b. Desde esta perspectiva, el aprendizaje cooperativo representa una forma de gestión social del aula, que no solamente aporta en lo académico, sino que propicia paralelamente el surgimiento de otros saberes actitudinales, junto con normas y valores de carácter social y emocional, de gran interés de cara a la construcción de culturas de aula en donde imperen principios de equidad y convivencia social.

En lo concerniente al tercer objetivo, en el cual se plantea conocer los métodos de aprendizaje cooperativo más empleados por la investigación especializada (Johnson; Johnson; Holube, 1999); (Durán y Vidal 2004); (Topping, 2005); (Durán, 2014) y sus posibles usos en situaciones de enseñanza y aprendizaje, se concluye lo siguiente:

a. Es importante reconocer en este aspecto, la necesidad de hacer un uso deliberado, consciente y estratégico de las metodologías señaladas y evitar su uso meramente instrumental y desarticulado. Es decir, toda decisión con respecto al uso de ellas, requeriría de una planificación previa del docente sobre las condiciones de aula, tipos de competencias de los estudiantes y características 
particulares de los contenidos a enseñar. Lo anterior permite al docente realizar agrupamientos de estudiantes cada vez más afines al trabajo en equipo requerido y el empleo de métodos cada vez más ajustados a los diversos escenarios.

b. A partir de los principios del aprendizaje entre iguales y aprendizaje cooperativo desarrollados en este artículo, cualquier docente interesado en el tema podría hacer un uso combinado de estas metodologías o diseñar sus propias pautas de trabajo cooperativo en el aula, siempre y cuando estén sustentadas en los principios acá expuestos y dichas prácticas obedezcan a decisiones intencionales y estratégicas, que el docente ejecuta en función de los determinados contextos de enseñanza en los que participa.

\section{Referencias bibliográficas}

Cohen, P.A., Kulik, J.A., Y Kukik, C.C. (1982). Educational outcomes of tutoring: A meta-analysis of findings. American Educational Research Journal, 19: 237-248.

Colomina, R.; Onrubia, J. (2005). Interacción educativa y aprendizaje escolar: La interacción entre alumnos. En C. Coll, J. Palacios, y Á. Marchesi. (Comps), Desarrollo Psicológico y Educación II. Psicología de la Educación, 415-435. Madrid: Alianza.

Davison, N. (1994). Cooperative and Collaborative Learning. En Thousand, J.S.; Nevin, A. (eds.): Creative and Collaborative Learning. Baltimoure: Paul H. Brookes Publishing Company.

Damon, W.; Phelps, E. (1989). Critical distinctions among three approaches to peer education. International Journal of Educational Research, 13: 9-19.

Deutshc, M. (1949). "A Theory of Cooperation and Competition". Human Relations, 2: 129-152.

Durán, D.; Vidal, V. (2004). Tutoría entre iguales: De la teoría a la Práctica. Barcelona: Graó.

Durán, D. y Monereo, C. (2008). The Impact of Peer Tutoring on the Improvement of Linguistic Competence, Self-Concept as Writer and Pedagogical Satisfaction, School Psychology International, 29(4): 481-499. 
Durán, D. y Sánchez G. (2012). Ritmos en dos: Una experiencia basada en la tutoría entre iguales para la mejora de la fluidez y comprensión musical. ํ5 56, pp 99-106. Eufonía. Graó.

Durán, D. (2014). Aprenseñar: Evidencias e implicaciones educativas de aprender enseñando. Madrid, España: Narcea, S.A. Ediciones.

Echeita, G. y Martin, E. (1990). Interacción social y aprendizaje. En A. Marchesi, C.Coll y J. Palacios. (Eds.). Desarrollo psicológico y educación III. Necesidades educativas especiales y aprendizaje escolar. Madrid: Alianza Psicológica.

Fernández, F. (2007). La tutoría entre compañeros en la Universidad. Tesis doctoral no publicada. Universidad de Granada.

Ferreiro R.; Calderón, M. (2001). El ABC del Aprendizaje Cooperativo: Trabajo en equipo para enseñar y aprender. Distrito Federal: Trillas.

Grannot, N. (1993). Patterns of Interaction in the Co-Construction of Knowledge: Separate Minds, Joint Effort, and Weird Creatures', En R. Wosniak and K. Fischer (eds) Development in Context. Acting and Thinking in Specific Environments, 74-91. Hillsdale, NJ: LEA.

Hairul, I.; Joyce, A. (2005). Learning Within Scripted and Nonscripted Peer-Tutoring Sessions: The Malaysian Context, The Journal of Educational Research, 99 (2): 67-77.

Johnson, D.W; Johnson, R.T; Holube, E. (1999). El Aprendizaje Cooperativo en el Aula. Buenos Aries: Paidós.

Melero, M.A.; Fernandez, P. (1995). El aprendizaje entre iguales: el estado de la cuestión en Estados Unidos, En Fernández, P.; Melero, M.A (comps.): La interacción social en contextos educativos. Madrid: Siglo XXI.

Monereo, C.; Pozo, I.; Castelló, M. (2001). La enseñanza de estrategias de aprendizaje en el contexto escolar, En C. Coll, J. Palacios, y Á. Marchesi. (Comps), Desarrollo Psicológico y Educación, II. Psicología de la Educación, 235-258. Madrid: Alianza.

Murray, F. (2001). Why Understanding the Theoretical Basis of Cooperative Learning Enhances Teaching Success, in J. Thousand, R. Villa and A. Nevin (eds) Creativity and Collaborative Learning, 3-13. Baltimore, MD: Paul H. Brookes Publishing. 
Ovejero, A. (1991). El aprendizaje Cooperativo. Una alternativa eficaz a la enseñanza tradicional. Barcelona: Promociones y Publicaciones Universitarias.

Palincsar, A. y Brown, A. (1984). Reciprocal teaching of comprehension-fostering and metacognitive strategies, Cognition and instruction, 1: 117-175.

Pujolás, P. (2003). Aprendre junts alumnes diferents. Els equips d'aprenentatge cooperatiu a l'aula. Vic, Barcelona: Eumo.

Pujolás, P. y Lago, J.R. (2007). La organización cooperativa de la actividad educativa. En Bonals, J. y Sánchez, M. (Coords) Manual de asesoramiento psicopedagógico, 349-390. Barcelona. Graó.

Sánchez, G. (2009). Efecto del Programa de Tutoría entre Iguales "Ritmos en Dos" en el desarrollo de Competencias de Lectura Rítmica Musical. Tesis doctoral. Facultad de Psicología. Universidad Autónoma de Barcelona.

Sharan, S. (ed.) (1992). Handbook of cooperative learning methods. London: Praeger.

Slavin, R.E (1977). A student team approach to teaching adolescents with special emotional and behavioral needs, Psychology in the Schools, 14(1): 77-84.

Topping, K. (1996). Effective Peer Tutoring in Further and Higher Education. Birmingham: SEDA paper.

Topping, K. (2005). Trends in Peer Learning, Educational Psychology 25(6): 631-645.

Wertsch, J.V. (1988). Vygotsky y la Formación Social de la Mente, Barcelona: Paidós. 